\title{
MACROPHAGE MIGRATION INHIBITORY FACTOR: A REGULATOR OF INNATE IMMUNITY
}

\section{Thierry Calandra and Thierry Roger}

For more than a quarter of a century, macrophage migration inhibitory factor (MIF) has been a mysterious cytokine. In recent years, MIF has assumed an important role as a pivotal regulator of innate immunity. MIF is an integral component of the host antimicrobial alarm system and stress response that promotes the pro-inflammatory functions of immune cells. A rapidly increasing amount of literature indicates that MIF is implicated in the pathogenesis of sepsis, and inflammatory and autoimmune diseases, suggesting that MIF-directed therapies might offer new treatment opportunities for human diseases in the future.

TOLL-LIKE RECEPTORS (TLRs). A family of receptors that recognize conserved products unique to microorganisms (such as lipopolysaccharide). Stimulation through TLRs induces dendriticcell maturation and activation, leading to optimal activation of the adaptive immune response. TLR-mediated events signal to the host that a microbial pathogen is present.
Division of Infectious Diseases, Department of Internal Medicine, Centre Hospitalier Universitaire Vaudois, $\mathrm{CH}-1011$

Lausanne, Switzerland. Correspondence to T. C. e-mail:Thierry.Calandra@ hospvd.ch

doi:10.1038/nri1200
Sensing and eliminating invading microorganisms is required for the survival for all living creatures. Genetic defects resulting in a failure to distinguish between self and non-self are life-threatening, as they predispose affected individuals to unrestricted microbial invasion and overwhelming infections. Natural host defences against microorganisms are mediated by the innate immune system - the most ancient of the two branches of the immune system ${ }^{1-3}$. The detection of pathogens is first carried out by sentinel cells of the innate immune system, such as macrophages and dendritic cells, located in tissues that are in contact with the host's natural environment, and then by circulating granulocytes and monocytes that are rapidly recruited to the site of infection. This process involves coordinated actions of the alternative and mannose-binding lectin pathways of complement, of acute-phase proteins and of germ-line-encoded pattern-recognition receptors, such as CD14 and TOLL-LIKE RECEPTORS (TLRs). Binding of conserved microbial molecular motifs, often known as pathogen-associated molecular patterns (PAMPs), to these receptors activates signaltransduction pathways and the transcription of immune genes, resulting in the release of numerous immunoregulatory molecules into the extracellular milieu and the expression of co-stimulatory molecules at the cell surface.
Among these effector molecules, cytokines have an essential role as they initiate the host inflammatory response and coordinate the cellular and humoral responses, which lead, within minutes to a few hours, to either the eradication or the containment of the invasive pathogen. The increased susceptibility to infection of animals in which genes encoding cytokines or cytokine receptors are mutated or deleted is strong evidence to support a crucial role for cytokine- or cytokine-receptor-dependent signalling pathways in regulating antimicrobial host defences. However, dysregulated production of pro-inflammatory mediators might also become life-threatening, as shown in patients with severe sepsis or septic shock, indicating that tight regulation of cytokine production is required.

Discovered almost 40 years ago during studies of the DELAYED-TYPE HYPERSENSITIVITY reaction ${ }^{4,5}$, macrophage migration inhibitory factor (MIF) was one of the first cytokines to be identified (TIMELINE). However, authentic biological activities of MIF remained ambiguous until the cloning of human MIF complementary DNA in 1989 (REF. 6). In 1991, a search for new regulators of inflammation led to the re-discovery of MIF as a molecule released, similar to a hormone, by cells of the anterior pituitary gland after exposure to the endotoxin lipopolysaccharide (LPS) ${ }^{7}$. This intriguing observation indicated that MIF could be a mediator that links the 
Timeline | The history of MIF

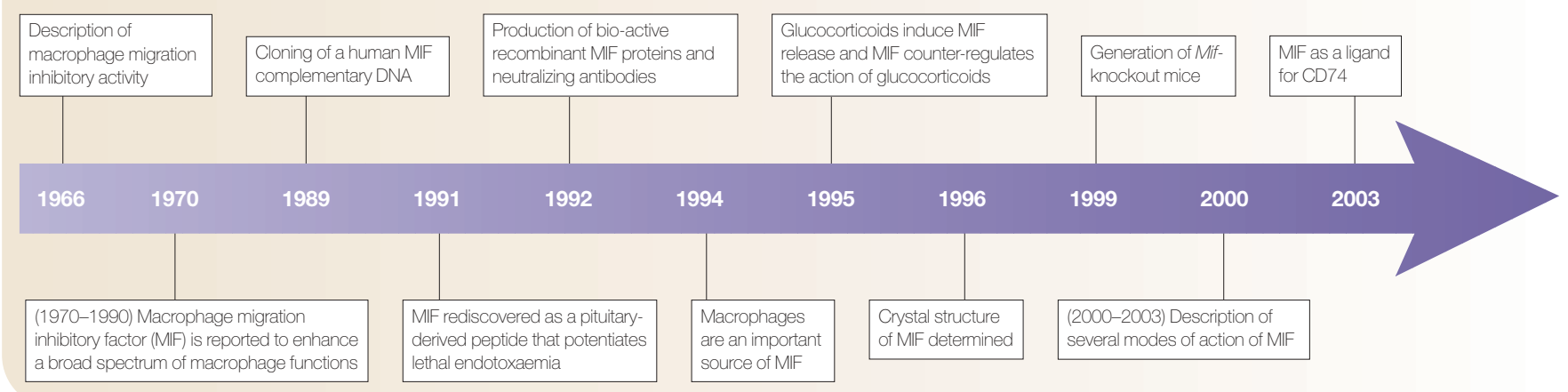

endocrine and immune systems. Mif-knockout mice were generated in 1999, and reported to be healthy and devoid of any apparent deficit ${ }^{8}$. In this article, we review the main features and biological activities of MIF. Special emphasis is placed on the emerging concept that MIF has a central role as a regulator of innate immune and inflammatory responses, and the implications it might have for the development of new therapies for human sepsis and other inflammatory diseases.

\section{Structure of the MIF gene and protein}

There is only one MIF gene in the human genome located on chromosome 22 (22q11.2), which is in SYNTENIC CONSERVATION with part of the mouse chromosome 10 that contains the Mifgene $e^{6,9-13}$. At least nine Mif pseudogenes have been found in the mouse genome $^{12,13}$. Additional information on the structure of the human MIF gene is provided in FIG. 1. Searching of the human genome for homologues of MIF indicated that $\mathrm{D}$-dopachrome tautomerase $(D D T)$ was the only gene with marked homology to $M I F^{14}$. As both genes are located relatively close on chromosome 22 , it is tempting to speculate that the MIF and DDT genes are duplications of a common ancestral gene that have evolved to have different biological functions. Two polymorphisms of the human MIF gene have been linked to human diseases. One is a single-nucleotide mutation (a G-to-C transition at position -173) in the $5^{\prime}$ flanking region, which is associated with systemic-onset juvenile arthritis ${ }^{15}$. The other polymorphism is a CATT-tetranucleotide repeat at position -794, which correlates with disease severity in a cohort of patients with rheumatoid arthritis ${ }^{16}$.

A single MIF messenger RNA species of $\sim 0.8 \mathrm{~kb}$ is found in humans, mice and rats. It encodes a 114amino-acid non-glycosylated protein of $12.5 \mathrm{kDa}$. All mammalian MIFs (human, mouse, rat and cattle) have $\sim 90 \%$ homology. MIF, also known as glycosylationinhibiting factor (GIF), has been reported to suppress IgE synthesis and have antigen-specific suppressor activity ${ }^{17}$. Surprisingly, MIF does not seem to belong to any cytokine superfamily. Homologues of mammalian MIF have been found in chickens, both jawless and jawed fish, ticks, parasites, plants (Arabidopsis thaliana) and cyanobacteria ${ }^{9,18-21}$. Conservation of MIF across species indicates that MIF might have important biological functions.
Crystallographic studies of human and rat MIFs indicate that MIF is a homotrimer (FIG. 2). There is marked three-dimensional structural homology between MIF and three microbial enzymes - oxalocrotonate tautomerase, 5-carboxymethyl-2-hydroxymuconate isomerase and chorismate mutase ${ }^{22-26}$. MIF can mediate DTT, phenylpyruvate keto-enol isomerase and thiol-protein oxidoreductase activity ${ }^{26-32}$. The amino-terminal proline residue is crucial for the catalytic activity; however, it is unclear whether a functional enzyme activity of MIF is required for its biological function ${ }^{33-36}$. It is possible that the enzyme activities of MIF represent vestigial signatures of the common ancestral origin of MIF and DDT genes. Yet, the fact that the amino-terminal proline residue, for example, has been conserved through evolution is evidence against such an hypothesis.

Expression patterns of MIF. At first, T cells were thought to be the main cellular source of MIF in the immune system. However, monocytes, macrophages, blood dendritic cells, B cells, neutrophils, eosinophils, mast cells and basophils have since been shown to express MIF $^{37-39}$. In contrast to most cytokines, MIF is constitutively expressed and stored in intracellular pools, and therefore does not require de novo protein synthesis before secretion. Similar to interleukin-1 (IL-1), basic fibroblast growth factor and a secreted form of cyclophilin, MIF lacks an amino-terminal leader sequence, indicating that it is released from cells by a non-conventional protein-secretion pathway. Besides the immune system, MIF has a broad tissue distribution (FIG. 3). Notably, MIF is expressed by cells and tissues that are in direct contact with the host's natural environment, such as the lung, the epithelial lining of the skin, and gastrointestinal and genitourinary tracts. Another distinctive feature of MIF is its high level of expression by several tissues of the endocrine system, especially by organs that are involved in stress responses (hypothalamus, pituitary and adrenal glands $)^{7,40-42}$.

\section{Mode of action of MIF}

MIF receptor. Cytokines act by binding to cognate receptors expressed by target cells, thereby activating signal-transduction pathways, gene transcription and the expression of downstream effector molecules. In support of a mode of action mediated by the interaction 
CD74

CD74, also known as the MHC

class-II-associated invariant

chain (Ii), is implicated in the

transport of MHC class II

proteins from the endoplasmic

reticulum to the Golgi complex.

About $5 \%$ of the cellular conten

of CD74 is expressed at the cell

surface independently of MHC

class II molecules. The

intracellular domain of CD74

does not seem to contain

sequences that are known to

interact with signalling

molecules.

T HELPER $1 / 2$ CELLS

$\left(\mathrm{T}_{\mathrm{H}} 1 / \mathrm{T}_{\mathrm{H}} 2\right)$. Subsets of CD4

$\mathrm{T}$ cells that are characterized by

distinctive profiles of cytokine

expression. $\mathrm{T}_{\mathrm{H}} 1$ cells typically

produce interleukin-2 (IL-2),

IL-1 2 and interferon- $\gamma$ that

support macrophage activation

and the development of a

cellular-based immune response,

whereas $\mathrm{T}_{\mathrm{H}} 2$ cells typically

produce IL-4, IL-5 and IL-13

that drive a humoral-based

immune response. with a typical cytokine receptor, MIF was found to activate extracellular signal-regulated kinase 1 (ERK1)/ERK2 - members of the family of mitogen-activated protein kinases (MAPKs) (see next paragraph). MIF has been reported to bind to the extracellular domain of $\mathrm{CD} 74$ the cell-surface form of the MHC class-II-associated invariant chain ${ }^{43}$ (FIG. 4a). Activation of ERK1/ERK2, cell proliferation and the production of prostaglandin $\mathrm{E}_{2}$ $\left(\mathrm{PGE}_{2}\right)$ are activities of MIF that require the involvement of CD74. Whether CD74 is the long sought after MIF receptor or a docking molecule that is implicated in the presentation of MIF to its as-yet-unidentified receptor is unclear at present. The fact that the intracellular domain of CD74 does not seem to contain motifs that might interact with signal-transducing molecules would support the latter possibility.

MIF activates ERK1/ERK2 signalling. Studies of intracellular signalling events and proliferation of MIFstimulated quiescent fibroblasts showed that MIF induces rapid (within 30 minutes) and sustained (up to 24 hours) phosphorylation and activation of the ERK1-ERK2-MAPK pathway and cell proliferation ${ }^{44}$ (FIG. 4a). MIF-induced ERK1/ERK2 activation was protein kinase A dependent and associated with increased cytoplasmic phospholipase A2 (PLA2) enzyme activity. PLA2 is an important intracellular link in the activation of the pro-inflammatory cascade, resulting first in the production of arachidonic acid and then of prostaglandins and leukotrienes. PLA2 also is a key target of the anti-inflammatory effects of glucocorticoids, and the ERK1/ERK2-mediated induction of PLA2 is one mechanism whereby MIF could override the immunosuppressive effects of steroids ${ }^{44}$ (see the section on MIF and inflammation).

MIF upregulates TLR4 expression. Constitutive expression of a cytokine by macrophages is unusual and intriguing, prompting the question of what kind of advantage does high baseline expression of MIF confer on macrophages? Studies carried out with macrophages transfected with antisense MIF constructs and macrophages that were isolated from Mif-deficient mice provided an answer to this question. Indeed, Mif-deficient macrophages were found to be hyporesponsive to LPS and Gram-negative bacteria, but not to other stimuli, as shown by reduced cytokine production due to the downregulation of expression of TLR4 - the signaltransducing molecule of the LPS receptor complex ${ }^{45,46}$. MIF upregulates the expression of TLR4 by acting on the ETS family of transcription factors, including PU.1, which are crucial for transcription of the mouse Tlr4 gene (FIG. 4a). Therefore, MIF facilitates the detection of endotoxin-containing bacteria, enabling cells that are at the forefront of the host antimicrobial defence system, such as macrophages, to respond rapidly to invasive bacteria. Rapid production of pro-inflammatory cytokines is absolutely essential for mounting the host defensive response. Consistent with this concept, it was reported recently that Mif-deficient mice failed to control the growth of the intracellular pathogen Salmonella typhimurium and succumbed to infection ${ }^{47}$. Increased susceptibility of Mif-deficient mice to infection was associated with reduced plasma levels of the cytokines tumour-necrosis factor (TNF), IL-12 and interferon- $\gamma$ (IFN- $\gamma$ ), but not of nitric oxide (NO), and with higher bacterial counts compared with wild-type mice. This indicates that MIF promotes a protective THELPER $1\left(\mathrm{~T}_{\mathrm{H}} 1\right)$-CELL immune response against $S$. typhimurium. Taken together, these observations provide a rationale for the constitutive expression of MIF by cells and tissues that are in close proximity to the external environment (FIG. 3). Moreover, it offers a mechanism whereby Mif-deficient mice are resistant to lethal endotoxaemia ${ }^{8}$.

MIF suppresses p53 activity. Primary tumours and numerous tumour-cell lines express high quantities of $\mathrm{MIF}^{48}$. A recent report indicating that MIF functions as a negative regulator of $\mathrm{p} 53$-mediated growth arrest and apoptosis has provided an interesting link between MIF, inflammation, cell growth and tumorigenesis ${ }^{49}$. Following this observation, it was reported that the proinflammatory function (that is, the production of TNF, IL-1 $\beta$ and PGE $_{2}$ ) and the viability of MIF-deficient macrophages were reduced compared with wild-type cells after challenge with LPS ${ }^{50}$. Despite the equal level of production of NO by MIF-deficient and wildtype macrophages, NO was thought to be a crucial mediator of increased apoptosis of MIF-deficient macrophages stimulated with LPS. Indeed, MIF was found to inhibit NO-induced intracellular accumulation of p53 and, therefore, p53-mediated apoptosis (FIG. 4b). Inhibition of $\mathrm{p} 53$ by MIF required serial activation of ERK1/ERK2, PLA2, cyclooxygenase 2 (COX2) and PGE 2 .

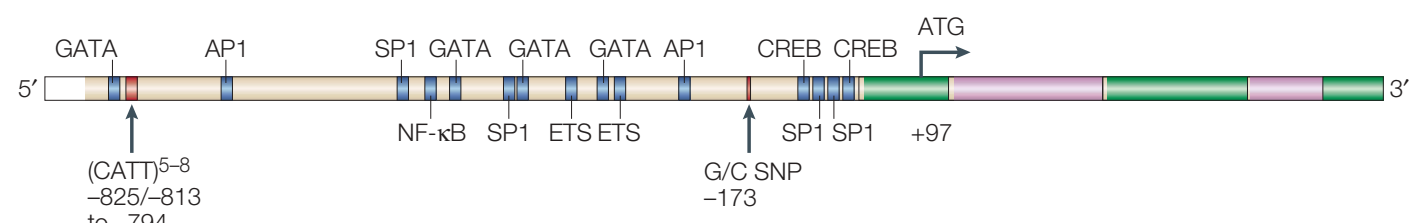

Figure 1 | Structure of the human MIF gene. The human macrophage migration inhibitory factor (MIF) gene is composed of three short exons (green boxes) of 107, 172 and 66 base pairs, and two introns (pink boxes) of 188 and 94 base pairs. Its 5' regulatory region contains several consensus DNA-binding sequences for transcription factors, notably activator protein 1 (AP1), nuclear factor- $\mathrm{kB}$ (NF-kB), ETS, GATA, SP1 and CAMP response element binding protein (CREB). However, little is known about the relevance of these putative DNA-binding sites in the regulation of expression of the human MIF gene. Two polymorphisms of the human MIF gene (arrows) - a CATT-tetranucleotide sequence repeated five to eight times at position -794 and a G-to-C single nucleotide polymorphism (SNP) at position -173 - have been associated with the severity of rheumatoid arthritis and systemic-onset juvenile idiopathic arthritis. 
YEAST TWO-HYBRID SYSTEM A screening system for protein-protein interactions, which results in the transcription of a reporter gene when a 'bait' protein that is attached to a DNA-binding domain comes into contact with a 'prey' protein bound to a transcriptional activator.

\section{ENDOCYTOSIS}

A process whereby extracellular material is internalized by a cell, which can occur either in a receptor-independent and often non-specific manner (for example, by pinocytosis) or in a receptor-dependent manner. Both clathrin-dependent endocytosis and clathrinindependent internalization triggered by lipid rafts can occur.
In agreement with these results, MIF was reported to interact with the $\mathrm{E} 2 \mathrm{~F}-\mathrm{p} 53$ pathway to sustain normal and malignant cell growth ${ }^{51}$. Therefore, the upregulation of expression of TLR4 and sustained cell survival are two mechanisms whereby MIF promotes pro-inflammatory innate immune responses ${ }^{50,52}$.

MIF inhibits JAB1 activity. Using a YEAST TWO-HYBRID SYSTEM, an interaction between MIF and the protein known as JUN-activation domain-binding protein 1 (JAB1) or as COP9 signalosome subunit 5 (CSN5) was shown ${ }^{53}$. JAB1 activates JUN N-terminal kinase (JNK) to phosphorylate JUN and so functions as a co-activator of activator protein 1 (AP1) - a transcription factor that is implicated in cell growth, transformation and cell death ${ }^{54}$. Other functions ascribed to JAB1 include degradation of the cell-cycle inhibitor KIP1 and the tumour suppressor p53. MIF and JAB1 are co-localized in the cytoplasm and MIF inhibits the positive regulatory effects of JAB1 on the activity of JNK and AP1 (FIG. 4a).

This observation is intriguing for two other reasons. First, it indicates that cells can take up MIF by ENDOCYTOSIs. Given the abundant intracellular expression of MIF, it might be asked what advantage would the cell gain by using endocytosed MIF rather than intracellular MIF. Endocytosis of MIF might occur either in a receptor-dependent or receptor-independent manner. Therefore, one obvious difference might be that endocytosis of MIF induces cell activation, whereas unprocessed intracellular MIF would not. At first glance, endocytosis would seem to be an atypical mode of action for a classic cytokine. However, it does not necessarily imply that endocytosis of MIF would bypass the requirement for a direct interaction with a membrane-bound receptor. In fact, there are examples of endosomes being implicated in signal transduction that is mediated by ligand-activated cytokine receptors. One such example is the transforming growth factor- $\beta$ (TGF- $\beta$ ) receptor ${ }^{55-57}$. Second, the article by Kleemann et al. ${ }^{53}$ assigns growth-inhibitory and anti-inflammatory functions to MIF that go against what were thought to be two well-established properties of MIF - that is, its capacity to sustain cell growth and to induce proinflammatory responses. These apparently conflicting observations might be accounted for by differences in the concentrations of MIF or in the status (that is, quiescent or activated) of the cells that were used ${ }^{39,58}$.

\section{MIF and innate immunity}

Initially thought to be a T-cell cytokine of the adaptive immune system, MIF has emerged as a cytokine that has important functions in the innate immune system.

Endotoxins and Gram-negative bacteria. Endotoxins are a main virulence factor of Gram-negative bacteria ${ }^{59}$. When stimulated with LPS, macrophages release $\mathrm{MIF}^{40}$. Other pro-inflammatory effector molecules of immune cells, such as TNF and IFN- $\gamma$, are also strong inducers of MIF production by macrophages ${ }^{40}$. After it is released in the tissue or in the systemic circulation, MIF acts as a classic pro-inflammatory cytokine promoting innate and adaptive immune responses through the activation of macrophages and $\mathrm{T}$ cells.

Although MIF is required to combat infection (see the section on MIF and TLR4), high-level production of MIF is harmful during acute infections. Although MIF did not induce shock when injected alone, high doses of recombinant MIF exacerbated lethal endotoxaemia and Escherichia coli sepsis when co-injected with LPS or E. coli into mice ${ }^{7,60}$ (BOX 1). High tissue and circulating levels of MIF were detected in mice with sepsis, and neutralizing antibodies specific for MIF reduced the production of TNF and protected the mice from lethal endotoxic shock or sepsis induced by E. coli or CAECAL LIGATION AND PUNCTURE (CLP), even when treatment with MIF-specific antibody was started after the onset of bacterial peritonitis ${ }^{7,60}$. The ability to rescue animals from sepsis when treatment is given therapeutically and not prophylactically is important, as anti-sepsis therapy, by definition, is always administered after the onset of infection in humans. Recapitulating some of these findings, Mif-deficient mice were reported to be resistant to endotoxic shock ${ }^{8}$. Although Mif-deficient mice that were produced using a different Mif gene-targeting approach were first reported to be as sensitive as wildtype mice to LPS ${ }^{61}$, additional experiments carried out by another group of investigators seem to indicate that these Mif-deficient mice are also resistant to LPS (J. Nishihira, personal communication).
Figure 2 | Three-dimensional structural homology of MIF and bacterial isomerase.

Macrophage migration inhibitory factor (MIF; top and bottom left panels) is a trimer with structural homology with the bacterial isomerases 4-oxalocrotonate tautomerase (4-OT; top and bottom right panels) and 5-carboxymethyl-2-hydroxymuconate isomerase (CHMl; not shown). 


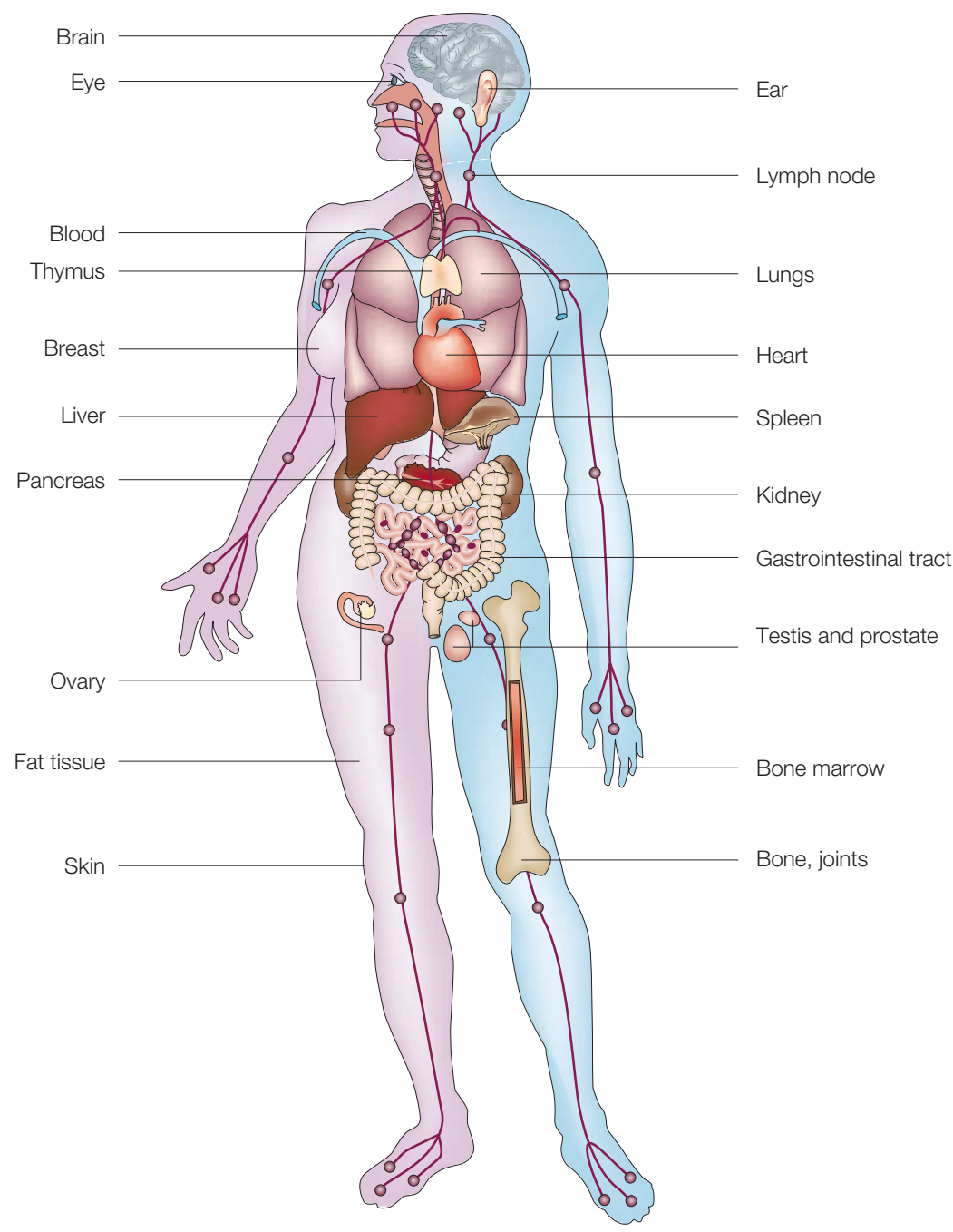

Figure 3 | Pattern of MIF expression. The tissue distribution and cellular sources of macrophage migration inhibitory factor (MIF) are shown. MIF is expressed in the brain (by the cortex, hypothalamus and cerebellum neurons, hippocampus, pons, glial cells, ependyma and astrocytes); in the eye (by the lens and epithelial cells of the cornea, iris and ciliary body, endothelial cells, and cells of the retina including epithelial cells, Muller cells and astrocytes); in the ear (by middle ear effusion); in the immune system (in the thymus, spleen, lymph nodes, blood and bone marrow, by monocytes/macrophages, T cells, B cells, dendritic cells, eosinophils, basophils, neutrophils and mast cells); in the lungs (by macrophages and epithelial cells); in the heart and vasculature (by endothelial cells); in the breast; in the endocrine system (by the pituitary gland, adrenal cortex and $\beta$-islet cells of the pancreas); in the liver (by Kuppfer cells, hepatocytes and endothelial cells); in the testis, prostate and ovaries (by Leydig cells, epithelial cells and granulosa cells of the follicles); in the gastrointestinal tract (by epithelial cells of the oesophagus, stomach, small and large intestines, and neurons); in the kidney (by epithelial cells, endothelial cells and mesangial cells); in fat tissue (by adipocytes); in the skin (by keratinocytes, sebaceous glands, hair follicles, endothelial cells and fibroblasts); and in bone and joints (by osteoblasts, fibroblasts and synoviocytes). toxin 1 (TSST1) or streptococcal pyrogenic exotoxin A (SPEA) induce the production of MIF by macrophages, which contributes to lethal pro-inflammatory responses. Mortality can be prevented by administration of neutralizing MIF-specific antibodies ${ }^{66}$ (BOX 1). Experiments carried out in Mif-deficient mice confirmed that a lack of Mif is associated with increased resistance to Grampositive shock caused by staphylococcal enterotoxin $\mathrm{B}^{8}$ (BOX 1). MIF is also released by cells of whole blood stimulated with heat-killed Streptococcus pneumoniae, and MIF-specific antibody reduces cytokine production and increases survival in a mouse model of S. pneumoniaeinduced pneumonia (T.C. et al., unpublished observations). Together with the observations obtained in experimental models of endotoxaemia and Gramnegative sepsis, these data indicate that MIF has an important role in the pathogenesis of bacterial infections.

MIF and host responses to other pathogens. In addition to bacterial sepsis, MIF has been implicated in the pathogenesis of parasitic (malaria, cysticercosis and leishmaniasis) and viral (cytomegalovirus and influenza virus) infections. MIF is produced in the lymph nodes of mice infected with Leishmania major, and in vivo administration of recombinant MIF reduced the severity of infection ${ }^{67}$. Mice that lack Mif were more susceptible to leishmaniasis and cysticercosis than wild-type mice ${ }^{68,69}$. Phagocytosis of erythrocytes infected with Plasmodium chabaudi or uptake of malarial pigment (hemozoin) by macrophages induced the release of $\mathrm{MIF}^{70}$. MIF inhibits erythroid, multipotential and granulocyte-macrophage progenitor-derived colony formation, indicating that it could be implicated in the pathophysiology of malarial anaemia. In pregnant women with placental malaria, the production of MIF by intervillous blood mononuclear cells is markedly upregulated ${ }^{71}$.

\section{MIF and inflammation}

A rapidly growing amount of evidence supports the notion that MIF is an integral component of host inflammatory responses. As summarized earlier, MIF is rapidly released by immune cells that are exposed to microbial products or to pro-inflammatory cytokines, or during antigen-specific activation, and has potent autocrine and paracrine effects that promote cell growth and survival. Using MIF-deficient cells, MIF-specific antibodies or recombinant MIF, investigators have provided evidence that MIF directly or indirectly promotes the production or expression of a large panel of proinflammatory molecules, including cytokines (such as TNF, IFN- $\gamma$, IL- $1 \beta$, IL-2, IL-6, IL-8 and macrophage inflammatory protein 2$)^{40,72-75}$, nitric oxide ${ }^{8,11}$, COX2 and products of the arachidonic acid pathway (such as $\left.\mathrm{PGE}_{2}\right)^{44,50}$, and several matrix metalloproteinases and their inhibitors ${ }^{76,77}$.

A surprising observation, which at first seemed to be incompatible with the pro-inflammatory features of this cytokine, was that MIF secretion was induced rather than inhibited by glucocorticoid hormones ${ }^{72}$. However, this paradoxical finding assisted in determining an 

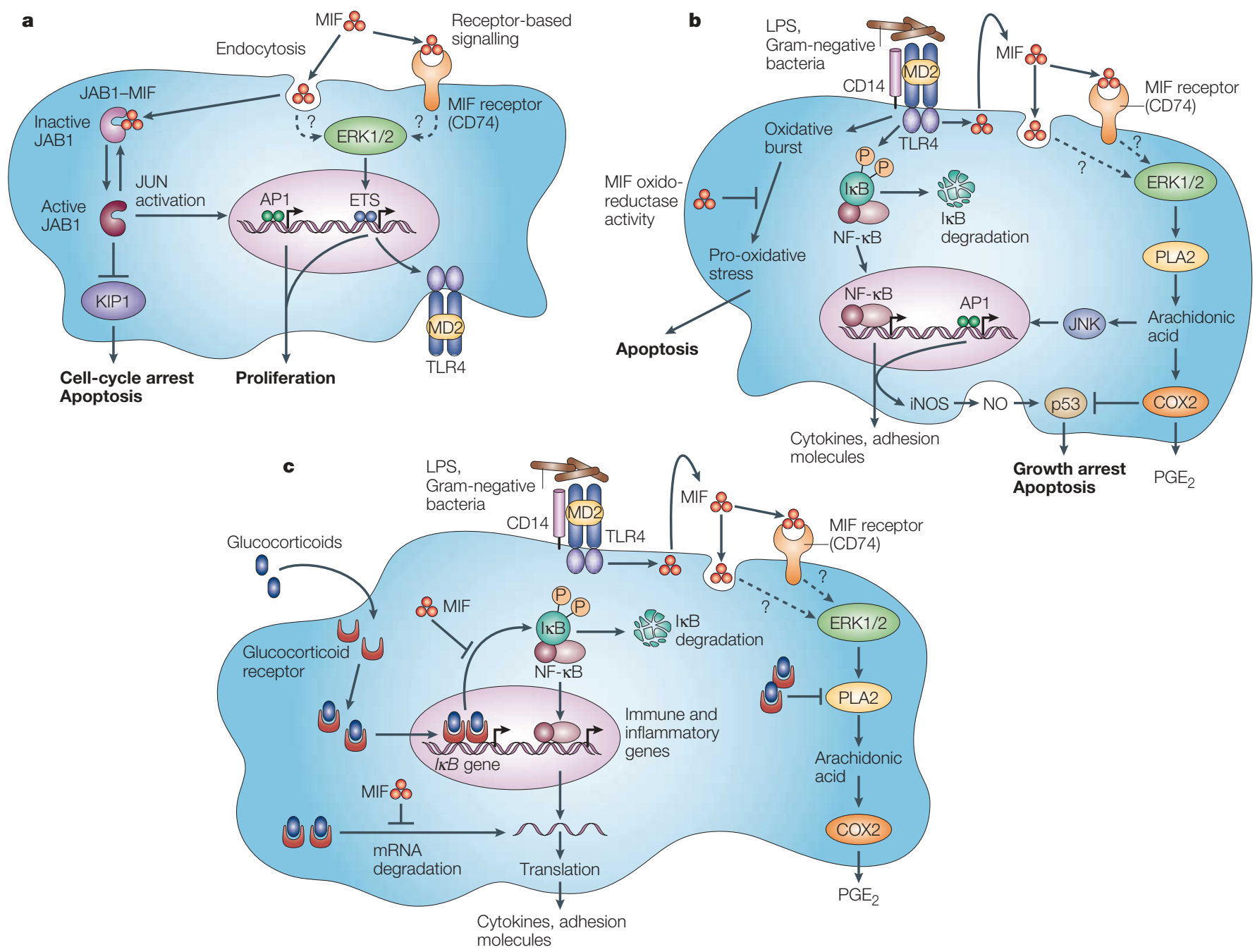

Apoptosis

CAECAL LIGATION AND

PUNCTURE

(CLP). An experimental model of infection that mimics human peritonitis. After laparotomy, the caecum is ligated distal to the ileocecal valve and punctured with a calibrated needle to produce leakage of faeces into the peritoneal cavity. The caecum is then returned to the peritoneal cavity and the abdomen is closed. CLP induces physiological changes that are similar to those seen in humans with spontaneous or post-surgical peritonitis.

MICROBIAL SUPERANTIGENS Molecules expressed by certain bacteria, viruses and mycoplasma that bind to the $\mathrm{V} \beta$-chain of the T-cell receptor and the MHC class II molecule of antigen-presenting cells, causing the activation of large subsets of $\mathrm{T}$ cells that express $\mathrm{V} \beta$-chains specific for the given superantigen.

Figure 4 | Mode of action of MIF. a | Macrophage migration inhibitory factor (MIF) might mediate its biological activities either through a classical receptor-mediated pathway or through a non-classical endocytic pathway. MIF has been shown to bind to CD74 and to phosphorylate the extracellular signal-regulated kinase 1 (ERK1)/ERK2 proteins. MIF promotes cell growth and activates transcription factors of the ETS family known to be crucial for expression of the Toll-like receptor 4 (TLR4) gene that encodes the signal-transducing molecule of the lipopolysaccharide (LPS) receptor complex. MIF binds to JUN-activation domain-binding protein 1 (JAB1), preventing JAB1-induced activation of JUN and JAB1-induced degradation of the cell-proliferation inhibitor KIP1, thereby leading to cell-cycle arrest and apoptosis. $\mathbf{b}$ | The induction and regulation of inflammatory responses of innate immune cells by MIF. MIF upregulates the expression of TLR4 by macrophages allowing rapid recognition of endotoxin-containing bacteria, which promotes the production of cytokines (including MIF), nitric oxide (NO) and other mediators. After it is released, MIF activates a cascade of events consisting of the phosphorylation of ERK1/ERK2, the induction of cytoplasmic phospholipase A2 (PLA2), arachidonic acid, JUN N-terminal kinase (JNK) activity and prostaglandin E2 ( $\left.\mathrm{PGE}_{2}\right)$. Through the generation of oxidoreductase activity and cyclooxygenase 2 (COX2), MIF prevents activation-induced apoptosis mediated by the oxidative burst and by p53. c | MIF counter-regulates the immunosuppressive effects of glucocorticoids at transcriptional and post-transcriptional levels. MIF inhibits the glucocorticoid-mediated induction of inhibitor of nuclear factor- $\kappa \mathrm{B}(\mathrm{l} \mathrm{\kappa B})$ synthesis and messenger RNA destabilization, and overrides the glucocorticoid-mediated inhibition of PLA2 activity and arachidonic acid production. iNOS, inducible nitric oxide synthase.

important feature of the biology of MIF. Indeed, MIF was found to override the immunosuppressive effects of glucocorticoids (FIG. 4c). In vitro, MIF was observed to reverse glucocorticoid-induced inhibition of TNF, IL-1, IL-6 and IL-8 synthesis by peripheral-blood mononuclear cells ${ }^{72}$, of cytosolic PLA2 activity, of arachidonicacid release by fibroblasts ${ }^{44}$ and of proliferation by $\mathrm{T}$ cells $\mathrm{s}^{73}$. This counter-regulatory effect of MIF was confirmed in mouse models of endotoxaemia ${ }^{72}$ and antigen-induced arthritis ${ }^{78}$. Similar to glucocorticoids, circulating concentrations of MIF are increased during inflammation, infection and stress ${ }^{60,72,79}$.

Analysis of the molecular mechanisms of MIF and glucocorticoid interactions have shown that MIF does interfere with glucocorticoids at a transcriptional and post-transcriptional level (FIG. 4c). Daun and Cannon ${ }^{80}$ showed that MIF antagonizes the effect of hydrocortisone on the nuclear factor- $\kappa \mathrm{B}(\mathrm{NF}-\kappa \mathrm{B})$-inhibitor of $\mathrm{NF}-\kappa \mathrm{K}(\mathrm{I} \kappa \mathrm{B})$ signal-transduction pathway by counteracting the steroid-mediated induction of cytosolic IKB $\alpha$. 
Confirming and extending these observations, we recently found evidence that MIF inhibits both the transcriptional and post-transcriptional regulation of cytokine production by glucocorticoids (T.C. and T.R., unpublished observations). Supported by increasing amounts of literature ${ }^{42,44,74,80,81}$, a concept has emerged that MIF acts as a physiological antagonist of

\section{Box $1 \mid$ Role of MIF in experimental models of sepsis}

Macrophage migration inhibitory factor (MIF) is required to combat infection caused by facultative intracellular pathogens. Compared with wild-type mice, Mif-deficient mice have impaired production of the cytokines tumour-necrosis factor (TNF), interferon- $\gamma$ (IFN- $\gamma$ ) and interleukin-12 (IL-12), resulting in a failure to eradicate Salmonella typhimurium and death from overwhelming sepsis (a). High levels of MIF expression are harmful during bacterial sepsis and toxic-shock syndromes. Administration of high doses of recombinant MIF to wild-type mice increases mortality in models of endotoxic (lipopolysaccharide, LPS-induced) shock and Escherichia coli sepsis (b). Mif-deficient mice are resistant to toxic shock induced by LPS or staphylococcal enterotoxin B (SEB) (c). MIF-specific antibody protects wild-type mice from lethal bacterial sepsis (peritonitis caused by E. coli or caecal ligation and puncture, CLP) and toxic-shock syndromes induced by LPS or staphylococcal toxic-shock syndrome toxin 1 (TSST1) (d). In the E. coli peritonitis model, improved survival is associated with reduced levels of TNF in the blood. Of note, mice with sepsis induced by CLP are protected even when treatment with MIF-specific antibody is delayed for up to 8 hours after the onset of infection. Therefore, in contrast to many other anti-cytokine therapies, treatment with MIF-specific antibody fulfills an important prerequisite for any anti-sepsis agent, that is the ability to rescue mice from death when treatment is given in a therapeutic manner (that is, once sepsis has developed). MIF-specific antibody also protects TNF-deficient mice from CLP, arguing for an intrinsic contribution of MIF to the pathogenesis of sepsis.
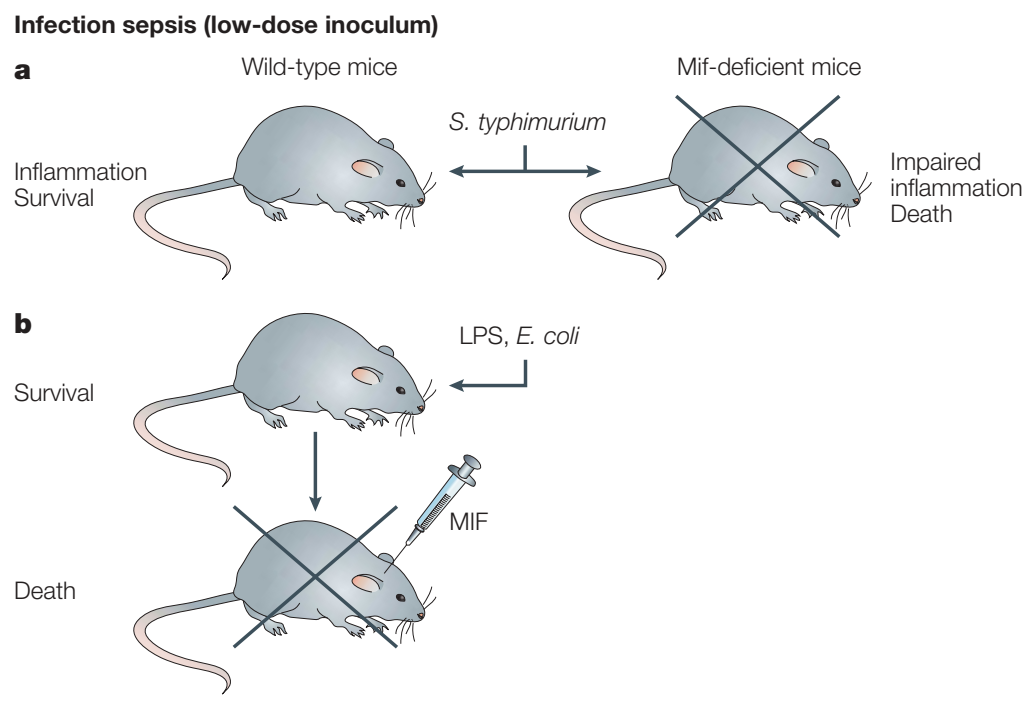

Severe sepsis (high-dose inoculum)

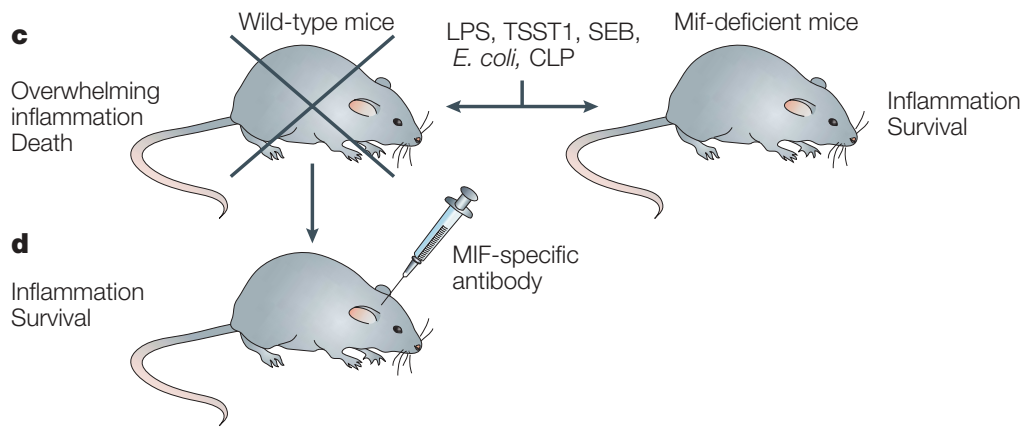

glucocorticoid activity, and that MIF and glucocorticoids function together to modulate innate and acquired immune responses. This concept has been further exemplified by recent studies implicating MIF in the pathogenesis of acute and chronic inflammatory and autoimmune diseases in humans and in experimental models (BOX 2).

\section{MIF and adaptive immunity}

Although MIF was discovered as a factor that is released by activated lymphocytes, so far little is known about its role in adaptive immunity. T cells constitutively express $\mathrm{MIF}^{73}$. Mitogens, tetanus toxoid, CD3-specific antibody, TSST1 and glucocorticoids have been reported to stimulate the release of MIF from T cells or from mouse splenocytes ${ }^{66,73,82}$. Although MIF is mainly thought to be produced by $\mathrm{T}_{\mathrm{H}} 2$ cells $\mathrm{s}^{73}$, it is also produced by $\mathrm{T}_{\mathrm{H}} 0$ and $\mathrm{T}_{\mathrm{H}} 1$ cells (T.C., unpublished observations). Possibly acting through an autocrine loop, MIF supports the activation and proliferation of $\mathrm{T}$ cells, and the production of IL-2 (REF. 73). MIF-specific antibodies prevented superantigen-induced activation and proliferation of splenocytes ${ }^{66}$, lending further support to the concept that MIF is also a lymphotropic cytokine. Moreover, MIF inhibits regulatory effects on cytotoxic CD8 ${ }^{+}$ $\mathrm{T}$ cells and regulates lymphocyte trafficking ${ }^{82}$. So, MIF also has important immunomodulatory functions in the adaptive immune system.

\section{Therapeutic implications}

MIF has been shown to be implicated in the pathogenesis of numerous acute and chronic inflammatory diseases ${ }^{39}$, including sepsis, acute respiratory distress syndrome (ARDS), asthma, arthritis, glomerulonephritis, inflammatory bowel diseases, atopic dermatitis, allograft rejection and most recently atherosclerosis (BOX 2). As the focus of this article is on innate immunity, we limit our discussion of the potential therapeutic implications of MIF to sepsis and ARDS.

Sepsis. Severe sepsis and septic shock are acute clinical manifestations of dysregulated innate immune responses. These life-threatening complications are the tenth most common cause of death in the United States and the second most common cause of death in noncoronary intensive-care units ${ }^{62}$. Over the past 20 years, considerable progress has been made in our understanding of the pathogenesis of sepsis ${ }^{65}$. Although a recent survey in the United States on the epidemiology of sepsis indicated that mortality is decreasing ${ }^{62}$, identification of new treatments is still a priority as death rates of severe sepsis (20-35\%) and septic shock (50-60\%) remain high.

The intrinsic pro-inflammatory properties of MIF, the fact that it is highly expressed by innate immune cells, and that it has a crucial role in macrophage responses against microbial products provide compelling evidence in support of a role for MIF in sepsis. This hypothesis has been tested in several experimental models of toxic shock and live bacterial sepsis, which have shown that excessive production of MIF is harmful in the acute 


\section{Box 2 | MIF in experimental models and human diseases}

Experimental models in which MIF is an important mediator of pathogenesis - Sepsis and toxic-shock syndrome ${ }^{7,8,60,66,72}$, delayed-type hypersensitivity ${ }^{88}$, adjuvantand antigen-induced arthritis ${ }^{89,90}$, glomerulonephritis ${ }^{91-94}$, acute lung injury ${ }^{75}$, allograft rejection ${ }^{95}$, inflammatory bowel disease (colitis) ${ }^{96,97}$, gastrititis ${ }^{98}$, pancreatitis ${ }^{99}$, atherogenesis ${ }^{100}$, encephalomyelitis ${ }^{101}$ and uveoretinitis ${ }^{102}$

Human pathologies associated with increased MIF expression by organs or systems - Immune system: sepsis, septic shock and allograft rejection ${ }^{60,79,83,84,103,104}$

- Lung: adult respiratory distress syndrome, asthma, tuberculosis and Wegener's granulomatosis ${ }^{4,85-87,105}$

- Kidney: glomerulonephritis ${ }^{106,107}$

- Bones and joints: rheumatoid arthritis, systemic-onset juvenile idiopathic arthritis and polychondritis $^{6,81,108-110}$

- Gastrointestinal tract: colitis and Crohn's disease ${ }^{96,111}$

- Skin: atopic dermatitis, psoriasis and systemic sclerosis ${ }^{112-114}$

- Endocrine system: type-2 diabetes and pancreatitis ${ }^{99,115}$

- Brain: multiple sclerosis and neuro-Behcet's disease ${ }^{116}$

- Eye: uveitis and iridocyclitis ${ }^{117,118}$

- Heart and vasculature: atherosclerosis ${ }^{119}$

- Ear: otitis ${ }^{120}$

MIF, macrophage migration inhibitory factor expression of MIF is upregulated in patients with ARDS and it diffuses into the alveolar air spaces, where it enhances alveolar inflammatory responses ${ }^{74}$. Immunoneutralization of MIF reduces the secretion of TNF and IL-8 in cultures of alveolar macrophages that are harvested from patients with ARDS. By contrast, addition of recombinant MIF enhances the release of TNF and IL-8 and so promotes the harmful pulmonary inflammatory response. As with peripheral-blood mononuclear cells and T cells, MIF was observed to counter-regulate the anti-inflammatory effects of glucocorticoids on cytokine secretion by cells of bronchoalveolar lavage fluid from patients with ARDS. In asthma - another model of acute lung inflammatory disease - stimulation of eosinophils with phorbol myristate acetate, C5a and IL-5 induced the immediate release of MIF. Bronchoalveolar lavage fluids and sputum from patients with asthma were found to contain increased levels of $\mathrm{MIF}^{86,87}$.

\section{Conclusions}

MIF has emerged recently as an important effector molecule of the innate immune system. Unlike most cytokines, MIF is expressed constitutively by immune and endocrine cells, and also by the epithelial lining of tissues in direct contact with the external environment, indicating a role for MIF as a regulator of host responses to infection and stress. Consistent with this hypothesis, MIF was found to upregulate the expression of TLR4 by macrophages - the signal-transducing molecule of the receptor complex for endotoxins of Gram-negative bacteria. Microbial products and pro-inflammatory cytokines induced the release of preformed MIF, which functions as a regulator of innate and adaptive immune responses. Distinctive features of MIF include its capacity to counter-regulate the immunosuppressive effects of glucocorticoids on immune cells and to sustain proinflammatory functions by inhibiting p53-dependent apoptosis of macrophages. By its pro-inflammatory and immunoregulatory properties, MIF contributes to the pathogenesis of severe sepsis, ARDS and autoimmune diseases, and high levels of MIF expression correlate with disease severity and poor outcome. Given the central place of MIF in the regulation of innate and acquired immunity, pharmacological or immunological modulation of MIF activity might offer new treatment options for patients with severe sepsis and inflammatory and autoimmune diseases.
1. Janeway, C. A. Jr. \& Medzhitov, R. Innate immune recognition. Annu. Rev. Immunol. 20, 197-216 (2002).

2. Hoffmann, J. A., Kafatos, F. C., Janeway, C. A. \& Ezekowitz, R. A. Phylogenetic perspectives in innate immunity. Science 284, 1313-1318 (1999)

3. Kimbrell, D. A. \& Beutler, B. The evolution and genetics of innate immunity. Nature Rev. Genet. 2, 256-267 (2001).

4. Bloom, B. R. \& Bennett, B. Mechanism of a reaction in vitro associated with delayed-type hypersensitivity. Science $\mathbf{1 5 3}$, 80-82 (1966).

5. David, J. R. Delayed hypersensitivity in vitro: its mediation by cell-free substances formed by lymphoid cell-antigen interaction. Proc. Natl Acad. Sci. USA 56, $72-77$ (1966). References 4 and 5 provided the first descriptions of macrophage migration inhibitory factor (MIF) activity.
6. Weiser, W. Y. et al. Molecular cloning of a cDNA encoding a human macrophage migration inhibitory factor. Proc. Natl Acad. Sci. USA 86, 7522-7526 (1989).

The first paper to report the cloning of human MIF complementary DNA.

7. Bernhagen, J. et al. MIF is a pituitary-derived cytokine that potentiates lethal endotoxaemia. Nature 365, 756-759 (1993). The rediscovery of MIF as a pro-inflammatory pituitary protein released in response to exposure to endotoxins.

8. Bozza, M. et al. Targeted disruption of migration inhibitory factor gene reveals its critical role in sepsis. J. Exp. Med. 189, 341-346 (1999).

The first description of Mif-knockout mice found to be resistant to bacterial endotoxin and staphylococcal exotoxin.
9. Wistow, G. J., Shaughnessy, M. P., Lee, D. C., Hodin, J. \& Zelenka, P. S. A macrophage migration inhibitory factor is expressed in the differentiating cells of the eye lens. Proc. Natl Acad. Sci. USA 90, 1272-1275 (1993).

10. Paralkar, V. \& Wistow, G. Cloning the human gene for macrophage migration inhibitory factor (MIF). Genomics 19, 48-51 (1994).

11. Bernhagen, J. et al. Purification, bioactivity, and secondary structure analysis of mouse and human macrophage migration inhibitory factor (MIF). Biochemistry $\mathbf{3 3}$, 14144-14155 (1994).

12. Kozak, C. A. et al. Genomic cloning of mouse MIF (macrophage inhibitory factor) and genetic mapping of the human and mouse expressed gene and nine mouse pseudogenes. Genomics 27, 405-411 (1995). 
13. Bozza, M. et al. Structural characterization and chromosomal location of the mouse macrophage migration inhibitory factor gene and pseudogenes. Genomics 27, 412-419 (1996).

14. Esumi, N. et al. Conserved gene structure and genomic linkage for D-dopachrome tautomerase $(D D T)$ and $M I F$. Mamm. Genome 9, 753-757 (1998).

15. Donn, R. P., Shelley, E., Ollier, W. E. \& Thomson, W. A nove $5^{\prime}$-flanking region polymorphism of macrophage migration inhibitory factor is associated with systemic-onset juvenile idiopathic arthritis. Arthritis Rheum. 44, 1782-1785 (2001).

16. Baugh, J. A. et al. A functional promoter polymorphism in the macrophage migration inhibitory factor (MIF) gene associated with disease severity in rheumatoid arthritis. Genes Immun. 3, 170-176 (2002).

17. Ishizaka, K., Ishii, Y., Nakano, T. \& Sugie, K. Biochemical basis of antigen-specific suppressor T cell factors: controversies and possible answers. Adv. Immunol. 74, $1-60$ (2000).

18. Sato, A. et al. Macrophage migration inhibitory factor (MIF) of jawed and jawless fishes: implications for its evolutionary origin. Dev. Comp. Immunol. 27, 401-412 (2003).

19. Jaworski, D. C., Jasinskas, A., Metz, C. N., Bucala, R. \& Barbour, A. G. Identification and characterization of a homologue of the pro-inflammatory cytokine macrophage migration inhibitory factor in the tick, Amblyomma americanum. Insect Mol. Biol. 10, 323-331 (2001).

20. Pastrana, D. V. et al. Filarial nematode parasites secrete a homologue of the human cytokine macrophage migration inhibitory factor. Infect. Immun. 66, 5955-5963 (1998).

21. Guiliano, D. B. et al. Conservation of long-range synteny and microsynteny between the genomes of two distantly related nematodes. Genome Biol. 3, RESEARCH0057 (2002).

22. Sun, H. W., Bernhagen, J., Bucala, R. \& Lolis, E. Crystal structure at 2. $6 \AA \AA$ resolution of human macrophage migration inhibitory factor. Proc. Nat/ Acad. Sci. USA 93, 5191-5196 (1996).

23. Suzuki, M. et al. Crystal structure of the macrophage migration inhibitory factor from rat liver. Nature Struct. Biol. 3, 259-266 (1996)

References 22 and 23 provided the first description of the three-dimensional structure of MIF, implying that MIF is a trimer with structural homology to bacterial isomerases.

24. Subramanya, H. S. et al. Enzymatic ketonization of 2-hydroxymuconate: specificity and mechanism investigated by the crystal structures of two isomerases. Biochemistry 95, 792-802 (1996).

25. Sugimoto, H. et al. Crystal structure of human D-dopachrome tautomerase, a homologue of macrophage migration inhibitory factor, at 1.54 A resolution. Biochemistry 38, 3268-3279 (1999).

26. Swope, M. D. \& Lolis, E. Macrophage migration inhibitory factor: cytokine, hormone, or enzyme? Rev. Physiol. Biochem. Pharmacol. 139, 1-32 (1999).

27. Rosengren, E. et al. The macrophage migration inhibitory factor MIF is a phenylpyruvate tautomerase. FEBS Letters 417, 85-88 (1997)

28. Rosengren, E. et al. The immunoregulatory mediator macrophage migration inhibitory factor (MIF) catalyzes a tautomerization reaction. Mol. Med. 2, 143-149 (1996). The first report of enzyme activity associated with MIF

29. Kleemann, R. et al. Disulfide analysis reveals a role for macrophage migration inhibitory factor (MIF) as thiol-protein oxidoreductase. J. Mol. Biol. 280, 85-102 (1998).

30. Kleemann, R., Kapurniotu, A., Mischke, R., Held, J. \& Bernhagen, J. Characterization of catalytic centre mutants of macrophage migration inhibitory factor (MIF) and comparison to Cys81Ser MIF. Eur. J. Biochem. 261, 753-766 (1999).

31. Nguyen, M. T. et al. The cytokine macrophage migration inhibitory factor reduces pro-oxidative stress-induced apoptosis. J. Immunol. 170, 3337-3347 (2003).

32. Lolis, E. \& Bucala, R. Macrophage migration inhibitory factor. Expert Opin. Ther. Targets 7, 153-164 (2003).

33. Bendrat, K. et al. Biochemical and mutational investigations of the enzymatic activity of macrophage migration inhibitor factor. Biochemistry 36, 15356-15362 (1997).

34. Hermanowski-Vosatka, A. et al. Enzymatically inactive macrophage migration inhibitory factor inhibits monocyte chemotaxis and random migration. Biochemistry $\mathbf{3 8}$ 12841-12849 (1999).

35. Kleemann, R. et al. Dissection of the enzymatic and immunologic functions of macrophage migration inhibitory factor. Full immunologic activity of $\mathrm{N}$-terminally truncated mutants. Eur. J. Biochem. 267, 7183-7193 (2000).

36. Lubetsky, J. B. et al. The tautomerase activity of MIF is a potential target for discovery of novel anti-inflammatory agents. J. Biol. Chem. 277, 24976-24982 (2002).
37. Martin, C., Roger, T. \& Calandra, T. Evolving Concepts in Sepsis and Septic Shock (eds Eichacker, P. Q. \& Pugin, J.) 45-67 (Kluwer Academic Publishers, Boston, Dordrecht, London, 2001).

38. Baugh, J. A. \& Bucala, R. Macrophage migration inhibiton factor. Crit. Care Med. 30, S27-S35 (2002)

39. Lue, H., Kleemann, R., Calandra, T., Roger, T. \& Bernhagen, J. Macrophage migration inhibitory factor (MIF): mechanisms of action and role in disease. Microbes Infect. 4, 449-460 (2002).

40. Calandra, T., Bernhagen, J., Mitchell, R. A. \& Bucala, R. The macrophage is an important and previously unrecognized source of macrophage migration inhibitory factor. J. Exp. Med. 179, 1895-1902 (1994)

Immune cells of the monocyte/macrophage lineage were found to be an abundant source of pre-formed MIF.

41. Bacher, M. et al. Migration inhibitory factor expression in experimentally induced endotoxemia. Amer. J. Pathol. 150 235-246 (1997)

42. Fingerle-Rowson, G. et al. Regulation of macrophage migration inhibitory factor expression by glucocorticoids in vivo. Am. J. Pathol. 162, 47-56 (2003).

43. Leng, L. et al. MIF signal transduction initiated by binding to CD74. J. Exp. Med. 197, 1467-1476 (2003). The authors describe the binding of MIF to the extracellular domain of CD74, also known as MHC class-II-associated invariant chain, leading to activation of the extracellular signal-regulated kinase 1 (ERK1)/ERK2 pathway.

44. Mitchell, R. A., Metz, C. N., Peng, T. \& Bucala, R. Sustained mitogen-activated protein kinase (MAPK) and cytoplasmic phopholipase A2 activation by macrophage migration inhibitory factor (MIF). J. Biol. Chem. 274, 18100-18106 (1999).

45. Roger, T., David, J., Glauser, M. P. \& Calandra, T. MIF regulates innate immune responses through modulation of Toll-like receptor 4. Nature 414, 920-924 (2001).

46. Roger, T., Froidevaux, C., Martin, C. \& Calandra, T. Macrophage migration inhibitory factor (MIF) regulates host responses to endotoxin through modulation of Toll-like receptor 4 (TLR4). J. Endotoxin Res. 9, 119-123 (2003).

47. Koebernick, H. et al. Macrophage migration inhibitory factor (MIF) plays a pivotal role in immunity against Salmonella typhimurium. Proc. Natl Acad. Sci. USA 99, 13681-13686 (2002).

48. Mitchell, R. A. \& Bucala, R. Tumor growth-promoting properties of macrophage migration inhibitory factor (MIF). Semin. Cancer Biol. 10, 359-366 (2000).

49. Hudson, J. D. et al. A proinflammatory cytokine inhibits p53 tumor suppressor activity. J. Exp. Med. 190, 1375-1382 (1999).

A report indicating that MIF functions as a negative regulator of p53-mediated growth arrest and apoptosis, providing an interesting link between MIF, inflammation, cell growth and tumorigenesis

50. Mitchell, R. A. et al. Macrophage migration inhibitory factor (MIF) sustains macrophage proinflammatory function by inhibiting p53: regulatory role in the innate immune response. Proc. Natl Acad. Sci USA 99, 345-350 (2002).

51. Petrenko, O., Fingerle-Rowson, G., Peng, T., Mitchell, R. A. \& Metz, C. N. Macrophage migration inhibitory factor deficiency is associated with altered cell growth and reduced susceptibility to Ras-mediated transformation. J. Biol. Chem. 278, 11078-11085 (2003).

52. Roger, T., Glauser, M. P. \& Calandra, T. Macrophage migration inhibitory factor (MIF) modulates innate immune responses induced by endotoxin and Gram-negative bacteria. J. Endotoxin. Res. 7, 456-460 (2001).

53. Kleemann, R. et al. Intracellular action of the cytokine MIF to modulate AP-1 activity and the cell cycle through Jab1. Nature 408, 211-216 (2000).

Report of a two-hybrid screen indicating the interaction between MIF and JUN-activation domainbinding protein 1 (JAB1) - a transcription factor implicated in cell growth, transformation and death. This report also indicates that cells might take up MIF by endocytosis.

54. Shaulian, E. \& Karin, M. AP-1 as a regulator of cell life and death. Nature Cell Biol. 4, E131-E136 (2002).

55. Tsukazaki, T., Chiang, T. A., Davison, A. F., Attisano, L. \& Wrana, J. L. SARA, a FYVE domain protein that recruits Smad2 to the TGF- $\beta$ receptor. Cell 95, 779-791 (1998).

56. Panopoulou, E. et al. Early endosomal regulation of Smaddependent signaling in endothelial cells. J. Biol. Chem. 277, 18046-18052 (2002).

57. Shi, Y. \& Massague, J. Mechanisms of TGF- $\beta$ signaling from cell membrane to the nucleus. Cell 113, 685-700 (2003).

58. Bucala, R. Signal transduction. A most interesting factor. Nature 408, 146-147 (2000).
59. Beutler, B. \& Rietschel, E. T. Innate immune sensing and its roots: the story of endotoxin. Nature Rev. Immunol. 3, 169-176 (2003)

60. Calandra, T. et al. Protection from septic shock by neutralization of macrophage migration inhibitory factor. Nature Med. 6. 164-170 (2000).

61. Honma, N. et al. Deficiency of the macrophage migration inhibitory factor gene has no significant effect on endotoxaemia. Immunol. 100, 84-90 (2000).

62. Martin, G. S., Mannino, D. M., Eaton, S. \& Moss, M. The epidemiology of sepsis in the United States from 1979 through 2000. N. Engl. J. Med. 348, 1546-1554 (2003).

63. Ikejima, T., Dinarello, C. A., Gill, M. \& Wolff, S. M. Induction of interleukin-1 by a product of Staphylococcus aureus associated with toxic shock syndrome. J. Clin. Invest. $\mathbf{7 3}$ 1312-1320 (1984).

64. Marrack, P. \& Kappler, J. The staphylococcal enterotoxins and their relatives. Science 248, 705-711 (1990).

65. Bochud, P. Y. \& Calandra, T. Pathogenesis of sepsis: new concepts and implications for future treatment. BMJ $\mathbf{3 2 6}$ 262-266 (2003).

66. Calandra, T., Spiegel, L. A., Metz, C. N. \& Bucala, R. Macrophage migration inhibitory factor is a critical mediator of the activation of immune cells by exotoxins of Grampositive bacteria. Proc. Natl Acad. Sci. USA 95 11383-11388 (1998)

67. Juttner, S. et al. Migration inhibitory factor induces killing of Leishmania major by macrophages: dependence on reactive nitrogen intermediates and endogenous TNF- $\alpha$. J. Immunol. 161, 2383-2390 (1998).

68. Satoskar, A. R., Bozza, M., Rodriguez, S. M., Lin, G. \& David, J. R. Migration-inhibitory factor gene-deficient mice are susceptible to cutaneous Leishmania major infection. Infect. Immun. 69, 906-911 (2001).

69. Rodriguez-Sosa, M. et al. Macrophage migration inhibitory factor plays a critical role in mediating protection against the helminth parasite Taenia crassiceps. Infect. Immun. 71, 1247-1254 (2003)

70. Martiney, J. A. et al. Macrophage migration inhibitory factor release by macrophages after ingestion of Plasmodium chabaudi-infected erythrocytes: possible role in the pathogenesis of malarial anemia. Infect. Immun. 68 2259-2267 (2000)

71. Chaisavaneeyakorn, S. et al. Immunity to placental malaria. IV. Placental malaria is associated with upregulation of macrophage migration inhibitory factor in intervillous blood. J. Infect. Dis. 186, 1371-1375 (2002).

72. Calandra, T. et al. MIF as a glucocorticoid-induced modulator of cytokine production. Nature 377, 68-71 (1995).

This paper describes the existence of a MIF-glucocorticoid counter-regulatory system that controls inflammatory and immune responses.

73. Bacher, M. et al. An essential regulatory role for macrophage migration inhibitory factor in T-cell activation. Proc. Nat Acad. Sci. USA 93, 7849-7854 (1996).

74. Donnelly, S. C. et al. Regulatory role for macrophage migration inhibitory factor in acute respiratory distress syndrome. Nature Med. 3, 320-323 (1997).

75. Makita, H. et al. Effect of anti-macrophage migration inhibitory factor antibody on lipopolysaccharide-induced pulmonary neutrophil accumulation. Am. J. Respir. Crit. Care Med. 158, 573-579 (1998).

76. Onodera, S. et al. High expression of macrophage migration inhibitory factor in the synovial tissues of rheumatoid joints. Cytokine 11, 163-167 (1999).

77. Onodera, S. et al. Macrophage migration inhibitory factor upregulates matrix metalloproteinase- 9 and -13 in rat osteoblasts. Relevance to intracellular signaling pathways. J. Biol. Chem. 277, 7865-7874 (2002).

78. Leech, M., Metz, C., Bucala, R. \& Morand, E. F. Regulation of macrophage migration inhibitory factor by endogenous glucocorticoids in rat adjuvant-induced arthritis. Arthritis Rheum. 43, 827-833 (2000).

79. Beishuizen, A., Thijs, L. G., Haanen, C. \& Vermes, I. Macrophage migration inhibitory factor and hypothalamo-pituitary-adrenal function during critical illness. J. Clin. Endocrinol. Metab. 86, 2811-2816 (2001).

80. Daun, J. M. \& Cannon, J. G. Macrophage migration inhibitory factor antagonizes hydrocortisone-induced increases in cytosolic lкB $\alpha$. Am. J. Physiol. Regul. Integr. Comp. Physiol. 279, R1043-R1049 (2000).

81. Leech, M. et al. Macrophage migration inhibitory factor in rheumatoid arthritis: evidence of proinflammatory function and regulation by glucocorticoids. Arthritis Rheum. 42, 1601-1608 (1999).

82. Abe, R., Peng, T., Sailors, J., Bucala, R. \& Metz, C. N. Regulation of the CTL response by macrophage migration inhibitory factor. J. Immunol. 166, 747-753 (2001).

83. Lehmann, L. E. et al. Plasma levels of macrophage migration inhibitory factor are elevated in patients with severe sepsis. Intensive Care Med. 27, 1412-1415 (2001). 
84. Gando, S. et al. Macrophage migration inhibitory factor is a critical mediator of systemic inflammatory response syndrome. Intensive Care Med. 27, 1187-1193 (2001)

85. Lai, K. N. et al. Role for macrophage migration inhibitory factor in acute respiratory distress syndrome. J. Pathol. 199 496-508 (2003).

86. Rossi, A. G. et al. Human circulating eosinophils secrete macrophage migration inhibitory factor (MIF). Potential role in asthma. J. Clin. Invest. 101, 2869-2874 (1998).

87. Yamaguchi, E. et al. Macrophage migration inhibitory factor (MIF) in bronchial asthma. Clin. Exp. Allergy 30, 1244-1249 (2000).

88. Bernhagen, J. et al. An essential role for macrophage migration inhibitory factor in the tuberculin delayed-type hypersensitivity reaction. J. Exp. Med. 183, 277-282 (1996).

89. Mikulowska, A., Metz, C. N., Bucala, R. \& Holmdahl, R. Macrophage migration inhibitory factor is involved in the pathogenesis of collagen type ll-induced arthritis in mice. J. Immunol. 158, 5514-5517 (1997)

90. Leech, M. et al. Involvement of macrophage migration inhibitory factor in the evolution of rat adjuvant arthritis. Arthritis Rheum. 41, 910-917 (1998).

91. Lan, H. Y. et al. De novo renal expression of macrophage migration inhibitory factor during the development of rat crescentic glomerulonephritis. Am. J. Pathol. 149 1119-1127 (1996).

92. Lan, H. Y. et al. The pathogenic role of macrophage migration inhibitory factor in immunologically induced kidney disease in the rat. J. Exp. Med. 185, 1455-1465 (1997).

93. Lan, H. Y. et al. TNF- $\alpha$ upregulates renal MIF expression in rat crescentic glomerulonephritis. Mol. Med. 3, 136-144 (1997).

94. Yang, N. et al. Reversal of established rat crescentic glomerulonephritis by blockade of macrophage migration inhibitory factor (MIF): potential role of MIF in regulating glucocorticoid production. Mol. Med. 4, 413-424 (1998).

95. Brown, F. G. et al. Upregulation of macrophage migration inhibitory factor in acute renal allograft rejection in the rat. Clin. Exp. Immunol. 118, 329-336 (1999).

96. de Jong, Y. P. et al. Development of chronic colitis is dependent on the cytokine MIF. Nature Immunol. 2, 1061-1066 (2001).

97. Ohkawara, T. et al. Amelioration of dextran sulfate sodium induced colitis by anti-macrophage migration inhibitory factor antibody in mice. Gastroenterol. 123, 256-270 (2002).

98. Huang, X. R. et al. Macrophage migration inhibitory facto is an important mediator in the pathogenesis of gastric inflammation in rats. Gastroenterol. 121, 619-630 (2001).
99. Sakai, Y. et al. Macrophage migration inhibitory factor is a critical mediator of severe acute pancreatitis. Gastroenterol. 124, 725-736 (2003).

100. Lin, S. G. et al. De novo expression of macrophage migration inhibitory factor in atherogenesis in rabbits. Circ. Res. 87, 1202-1208 (2000).

101. Denkinger, C. M., Denkinger, M., Kort, J. J., Metz, C. \& Forsthuber, T. G. In vivo blockade of macrophage migration inhibitory factor ameliorates acute experimental autoimmune encephalomyelitis by impairing the homing of encephalitogenic $T$ cells to the central nervous system. J. Immunol. 170, 1274-1282 (2003).

102. Kitaichi, N. et al. Inhibition of experimental autoimmune uveoretinitis with anti-macrophage migration inhibitory factor antibodies. Curr. Eye Res. 20, 109-114 (2000).

103. Joshi, P. C., Poole, G. V., Sachdev, V., Zhou, X. \& Jones, Q Trauma patients with positive cultures have higher levels of circulating macrophage migration inhibitory factor (MIF). Res. Commun. Mol. Pathol. Pharmacol. 107, 13-20 (2000).

104. Lan, H. Y. et al. Macrophage migration inhibitory factor expression in human renal allograft rejection. Transplantation 16, 1465-1471 (1998).

105. Yamada, G. et al. Elevated levels of serum macrophage migration inhibitory factor in patients with pulmonary tuberculosis. Clin. Immunol. 104, 123-127 (2002).

106. Lan, H. Y. et al. Expression of macrophage migration inhibitory factor in human glomerulonephritis. Kidney Int. 57, 499-509 (2000).

107. Matsumoto, K. \& Kanmatsuse, K. Increased production of macrophage migration inhibitory factor by $T$ cells in patients with IgA nephropathy. Am. J. Nephrol. 21, 455-464 (2001).

108. Morand, E. F. et al. Macrophage migration inhibitory factor in rheumatoid arthritis: clinical correlations. Rheumatol. $\mathbf{4 1}$ 558-562 (2002)

109. Meazza, C. et al. Macrophage migration inhibitory factor in patients with juvenile idiopathic arthritis. Arthritis Rheum. 46 232-237 (2002)

110. Ohwatari, R. et al. Serum level of macrophage migration inhibitory factor as a useful parameter of clinical course in patients with Wegener's granulomatosis and relapsing polychondritis. Ann. Otol. Rhinol. Laryngol. 110, 1035-1040 (2001).

111. Murakami, H., Akbar, S. M., Matsui, H. \& Onii, M. Macrophage migration inhibitory factor in the sera and at the colonic mucosa in patients with ulcerative colitis: clinical implications and pathogenic significance. Eur. J. Clin. Invest 31, 337-343 (2001)
112. Shimizu, T., Abe, R., Ohkawara, A., Mizue, Y. \& Nishihira, J. Macrophage migration inhibitory factor is an essential immunoregulatory cytokine in atopic dermatitis. Biochem. Biophys. Res. Commun. 240, 173-178 (1997).

113. Steinhoff, M. et al. Evidence for a role of macrophage migration inhibitory factor in psoriatic skin disease. $\mathrm{Br} . \mathrm{J}$. Dermatol. 141, 1061-1066 (1999).

114. Selvi, E. et al. Expression of macrophage migration inhibitory factor in diffuse systemic sclerosis. Ann. Rheum. Dis. 62 460-464 (2003).

115. Yabunaka, N. et al. Elevated serum content of macrophage migration inhibitory factor in patients with type 2 diabetes. Diabetes Care 23, 256-258 (2000).

116. Niino, M., Ogata, A., Kikuchi, S., Tashiro, K. \& Nishihira, J. Macrophage migration inhibitory factor in the cerebrospinal fluid of patients with conventional and optic-spinal forms of multiple sclerosis and neuro-Behcet's disease. J. Neurol. Sci. 179, 127-131 (2000).

117. Kitaichi, N. et al. Prominent increase of macrophage migration inhibitory factor in the sera of patients with uveitis. Invest. Ophthalmol. Vis. Sci. 40, 247-250 (1999).

118. Kitaichi, N. et al. Increase of macrophage migration inhibitory factor in sera of patients with iridocyclitis. Br. J. Ophthalmol. 84, 1423-1425 (2000).

119. Burger-Kentischer, A. et al. Expression of macrophage migration inhibitory factor in different stages of human atherosclerosis. Circulation 105, 1561-1566 (2002)

120. Kariya, S. et al. Role of macrophage migration inhibitory factor in otitis media with effusion in adults. Clin. Diagn. Lab. Immunol. 10, 417-422 (2003).

\section{Acknowledgements}

We thank M. Pagni for preparing figure 2 and J. Bernhagen for critical reading of the manuscript. The work was supported by the Swiss National Science Foundation, the Leenaards Foundation, the Santos Suarez Foundation and the Bristol-Myers Squibb Foundation. T. C. and T. R. are recipients of career and research awards of the Leenaards Foundation.

\section{(2) Online links}

\section{DATABASES}

The following terms in this article are linked online to:

LocusLink: http://www.ncbi.nlm.nih.gov/LocusLink/ CD74 | COX2 | DDT | IFN- $\gamma$ | IL-1 | IL-12 | JAB1 | KIP1 | MIF | p53 PLA2 | TLR4 | TNF

Access to this interactive links box is free online. 